\title{
Excluded-Volume Effects in Sodium Hyaluronate Solutions Revisited
}

\author{
Kikuko Tsutsumi and Takashi NorisuYe \\ Department of Macromolecular Science, Osaka University, \\ Toyonaka, Osaka 560-0043, Japan
}

(Received October 27, 1997)

\begin{abstract}
Five sodium salt samples of hyaluronic acid ranging in weight-average molecular weight $M_{w}$ from $1.9 \times 10^{5}$ to $1.5 \times 10^{6}$ have been studied by light scattering and viscometry in aqueous sodium chloride at $25^{\circ} \mathrm{C}$. The intrinsic viscosity $([\eta])$ data obtained at 10 different $\mathrm{NaCl}$ concentrations $C_{\mathrm{s}}$ covering a range from 0.005 to $2.5 \mathrm{M}$ are analyzed along with previous data for lower $M_{w}$ on the basis of the Yamakawa-Fujii-Yoshizaki theory for unperturbed wormlike chains combined with the quasi-two-parameter (QTP) theory for excluded-volume effects. Fairly satisfactory agreement is observed between theory and experiment at $C_{\mathrm{s}}$ higher than $0.01 \mathrm{M}$ over the range of molecular weight from $3.8 \times 10^{3}$ to $1.5 \times 10^{6}$, though the degree of agreement tends to lower with decreasing $C_{\mathrm{s}}$. When the wormlike chain parameters and the excluded-volume strength estimated from $[\eta]$ are used, the QTP theory with the known expression for $\left\langle S^{2}\right\rangle$ (the mean-square radius of gyration) of the unperturbed wormlike chain is found to describe almost quantitatively the measured $\left\langle S^{2}\right\rangle$ for Na hyaluronate at $C_{\mathrm{s}}=0.02$, 0.1 , and $0.5 \mathrm{M}$. Thus, it is concluded that this theory for nonionic chains is applicable, in a fairly good approximation, to $\left\langle S^{2}\right\rangle$ and $[\eta]$ of the charged polysaccharide in aqueous $\mathrm{NaCl}$ unless $C_{\mathrm{s}}$ is lower than $0.02 \mathrm{M}$.

KEY WORDS Hyaluronic Acid / Polysaccharide / Polyelectrolyte / Excluded-Volume Effect / Wormlike Chain /
\end{abstract}

According to recent experimental studies, ${ }^{1,2}$ the quasitwo-parameter (QTP) theory ${ }^{3}$ based on the wormlike ${ }^{4}$ or helical wormlike ${ }^{3}$ chain allows an almost quantitative, consistent description of excluded-volume effects on the mean-square radius of gyration $\left\langle S^{2}\right\rangle$ and the intrinsic viscosity $[\eta]$ for nonionic, linear polymers, both flexible and stiff. In contrast to this situation, our understanding of those effects in ionic chains still remains in a quite unsatisfactory stage.

In our previous work, Hayashi et al. ${ }^{5,6}$ examined, as a first step toward solving the electrostatic excludedvolume problem, the applicability of the QTP theory to $[\eta]$ of sodium hyaluronate, a charged linear polysaccharide with intrinsically weak stiffness, in aqueous sodium chloride with the aid of the Yamakawa-FujiiYoshizaki theory ${ }^{7,8}$ for unperturbed wormlike cylinders. The general agreement between theory and experiment was not bad over the entire range of weight-average molecular weight $M_{w}\left(3.8 \times 10^{3}<M_{w}<3.5 \times 10^{5}\right)$ and $\mathrm{NaCl}$ concentration $C_{\mathrm{s}}\left(0.005 \mathrm{M} \leq C_{\mathrm{s}} \leq 2.5 \mathrm{M}\right)$ studied, but at a few low $C_{\mathrm{s}}$ the experimental viscosity exponents appeared to exceed the theoretically predictable value in the region of $M_{w}>4 \times 10^{4}$ where excluded-volume effects were significant. Nonetheless, the discrepancies between the theoretical and experimental $[\eta]$ were too subtle to draw a definite conclusion on the applicability of the QTP scheme to Na hyaluronate. Thus it seemed necessary to extend the work to a higher molecular weight region where volume effects are more pronounced.

In the present study, viscosity data were obtained for five hyaluronate samples with $M_{w}$ of $1.9 \times 10^{5}$ to $1.5 \times 10^{6}$ in aqueous $\mathrm{NaCl}$ of different $C_{\mathrm{s}}$ and analyzed along with our previous data ${ }^{5,6}$ to check the applicability of the QTP scheme over a wider range of molecular weight. Radius of gyration data were also obtained from light scattering measurements at some $\mathrm{NaCl}$ concentrations. They were used for an independent check of the applicability.

\section{EXPERIMENTAL}

\section{Samples}

A hyaluronate sample with a purity higher than $99 \%$ and an intrinsic viscosity of $25.6 \times 10^{2} \mathrm{~cm}^{3} \mathrm{~g}^{-1}$ (in $0.2 \mathrm{M}$ aqueous $\mathrm{NaCl}$ at $25^{\circ} \mathrm{C}$ ) was supplied by Shiseido Co., Japan. Part of it was degraded to four different (average) molecular weights by heating at $110^{\circ} \mathrm{C}$ for $25-60 \mathrm{~min}$. The resulting samples (each 5-10 g) and the original one $(1.5 \mathrm{~g})$ were then fractionated with $0.2 \mathrm{M}$ aqueous $\mathrm{NaCl}$ as the solvent and acetone as the precipitant. The procedure was essentially the same as that employed previously, ${ }^{5}$ but to effect the fractionation, lower and higher molecular weight portions were repeatedly removed from each of the five samples and a small amount of the central portion $(0.2-0.5 \mathrm{~g})$ was extracted. The fractions thus obtained were designated as HAT1, HAT2 ,.., and HAT5 in order of increasing molecular weight.

Aqueous solutions of these fractions were passed through a mixed-bed ion exchanger (Amberlite IR$120+$ IR-400) and then neutralized with $0.1 \mathrm{~N}$ aqueous sodium hydroxide (see ref 5). Dry sodium hyaluronate was obtained by lyophilization of the neutralized solutions (for more than 4 days). It was dissolved directly in aqueous $\mathrm{NaCl}$ of a given $C_{\mathrm{s}}$ after being further dried in vacuo overnight.

\section{Light Scattering}

Scattering intensities were measured for the five $\mathrm{Na}$ salt samples in $0.02,0.1$, and $0.5 \mathrm{M}$ aqueous $\mathrm{NaCl}$ at $25^{\circ} \mathrm{C}$ on a Fica 50 light-scattering photometer in an angular range from 20 to $150^{\circ}$. Vertically polarized incident light of 436 or 546-nm wavelength was used. The experimental procedures including optical clarification were essentially the same as those described elsewhere. ${ }^{9}$ The intensity data obtained were analyzed by use of the square-root plots of $\left(K c / R_{\theta}\right)^{1 / 2} v s . c$ and $\left(K c / R_{\theta}\right)^{1 / 2} v s \cdot \sin ^{2}(\theta / 2)$ to evaluate $M_{w}, A_{2}$ (the second 
virial coefficient), and $\left\langle S^{2}\right\rangle_{z}$ (the $z$-average $\left\langle S^{2}\right\rangle$ ). Here, $K$ is the optical constant, $R_{\theta}$ the excess reduced scattering intensity at scattering angle $\theta$, and $c$ the polymer mass concentration.

The measurement was also made at $C_{\mathrm{s}}=0.005 \mathrm{M}$ for samples HAT1, HAT2, and HAT3. However, no reliable data for $M_{w}$ and $\left\langle S^{2}\right\rangle_{z}$ could be obtained, and our check of the QTP theory with $\left\langle S^{2}\right\rangle_{z}$ data was limited to $C_{\mathrm{s}} \geq 0.02 \mathrm{M}$.

The specific refractive index increments $(\partial n / \partial c)_{\mu}$ of $\mathrm{Na}$ hyaluronate in 0.02 and $0.1 \mathrm{M}$ aqueous $\mathrm{NaCl}$ at $25^{\circ} \mathrm{C}$ were determined for dialyzed solutions of a sample with [ $\eta$ ] (in $0.2 \mathrm{M}$ aqueous $\mathrm{NaCl}$ ) $=8.8 \times 10^{2} \mathrm{~cm}^{3} \mathrm{~g}^{-1}$ using a modified Schulz-Cantow type differential refractometer; the subscript $\mu$ attached to $(\partial n / \partial c)$ signifies the condition that the chemical potentials of all diffusible components are held constant. The values of $(\partial n / \partial c)_{\mu}$ obtained were $0.144 \mathrm{~cm}^{3} \mathrm{~g}^{-1}$ for $436 \mathrm{~nm}$ and $0.141 \mathrm{~cm}^{3} \mathrm{~g}^{-1}$ for $546 \mathrm{~nm}$ in both 0.02 and $0.1 \mathrm{M}$ aqueous $\mathrm{NaCl}$. For $(\partial n / \partial c)_{\mu}$ of $0.5 \mathrm{M} \mathrm{NaCl}$ solutions, the previous results ${ }^{5}(0.141$ and $0.138 \mathrm{~cm}^{3} \mathrm{~g}^{-1}$ for 436 and $546 \mathrm{~nm}$, respectively) were used.

\section{Viscometry}

Viscosities of aqueous $\mathrm{NaCl}$ solutions of $\mathrm{Na}$ hyaluronate at $25^{\circ} \mathrm{C}$ were measured at ten different salt concentrations ranging from 0.005 to $2.5 \mathrm{M}$ using a fourbulb low-shear capillary viscometer (apparent shear rates of $11-91 \mathrm{~s}^{-1}$ for pure water at $25^{\circ} \mathrm{C}$ ) or a conventional capillary viscometer of the Ubbelohde type (apparent shear rate of about $800 \mathrm{~s}^{-1}$ ). The apparent values of $[\eta]$ for a given sample, determined by the former viscometer, increased linearly with decreasing shear rate (in the above range) and were extrapolated to zero shear rate without ambiguity; we note that even for the highest molecular weight sample HAT5 in $0.005 \mathrm{M}$ aqueous $\mathrm{NaCl}$, the apparent $[\eta]$ value at the highest shear rate of $91 \mathrm{~s}^{-1}$ was only $6 \%$ smaller than the value at zero shear rate. No electroviscous effect on the measured $[\eta]$ was considered necessary for the reason mentioned previously. ${ }^{6}$ Huggins' constants were all in the range between 0.32 and 0.41 .

\section{RESULTS}

Figure 1 illustrates the light scattering envelopes for $\mathrm{Na}$ hyaluronate sample HAT5 in $0.1 \mathrm{M}$ aqueous $\mathrm{NaCl}$ at $25^{\circ} \mathrm{C}$, where the dashed line represents the initial tangent to the curve for $\left(K c / R_{\theta}\right)^{1 / 2}$ at infinite-dilution. The numerical data obtained from light scattering measurements are summarized in Table I. Two points are to be noted here. First, the values of $M_{w}$ for the respective samples at the three different salt concentrations agree within $\pm 2.1 \%$. Second, those of $A_{2}$ are all on the order $10^{-3} \mathrm{~mol} \mathrm{~cm}^{3} \mathrm{~g}^{-2}$, showing remarkable nonideality of hyaluronate solutions.

The relations between $\log \left\langle S^{2}\right\rangle_{z} v s . \log M_{w}$ at fixed $C_{\mathrm{s}}$ are shown in Figure 2. They are roughly linear with slopes of $1.18,1.20$, and 1.26 at $C_{\mathrm{s}}=0.5,0.1$, and $0.02 \mathrm{M}$, respectively. The last exponent slightly exceeds the asymptotic value of 1.20 for nonionic, flexible chains in good solvents. ${ }^{10}$

The data for zero-shear rate $[\eta]$ are summarized in
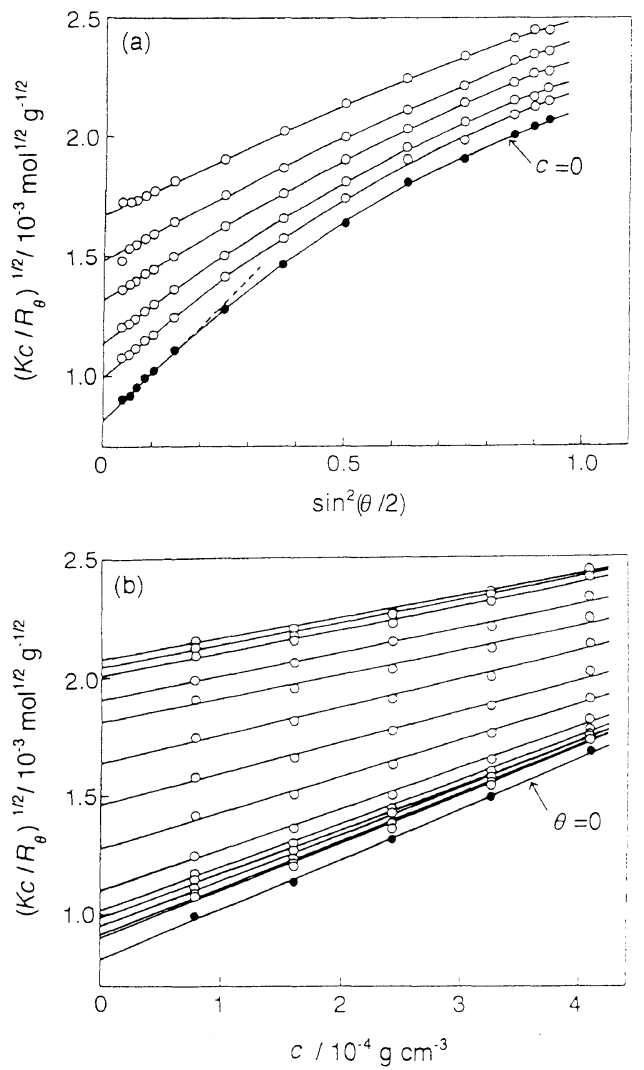

Figure 1. Light Scattering envelopes for $\mathrm{Na}$ hyaluronate sample HAT5 in $0.1 \mathrm{M}$ aqueous $\mathrm{NaCl}$ at $25^{\circ} \mathrm{C}$. The wavelength of incident light is $546 \mathrm{~nm}$.

Table I. Results from light scattering measurements on sodium hyaluronate samples in aqueous $\mathrm{NaCl}$ at $25^{\circ} \mathrm{C}$

\begin{tabular}{|c|c|c|c|c|}
\hline \multirow{2}{*}{ Sample } & $C_{\mathrm{s}}$ & \multirow{2}{*}{$10^{-5} M_{w}$} & $10^{3} A_{2}$ & \multirow{2}{*}{$\frac{10^{-4}\left\langle S^{2}\right\rangle}{\mathrm{nm}^{2}}$} \\
\hline & M & & $\mathrm{mol} \mathrm{cm}^{3} \mathrm{~g}^{-2}$ & \\
\hline \multirow[t]{3}{*}{ HAT1 } & 0.5 & 1.92 & 1.41 & 0.107 \\
\hline & 0.1 & 1.97 & 2.51 & 0.130 \\
\hline & 0.02 & 1.99 & 5.60 & 0.173 \\
\hline \multirow[t]{3}{*}{ HAT2 } & 0.5 & 2.50 & 1.35 & 0.144 \\
\hline & 0.1 & 2.50 & 2.50 & 0.167 \\
\hline & 0.02 & 2.45 & 5.41 & 0.219 \\
\hline \multirow[t]{3}{*}{ HAT3 } & 0.5 & 4.16 & 1.16 & 0.259 \\
\hline & 0.1 & 4.16 & 2.29 & 0.328 \\
\hline & 0.02 & 4.33 & 4.82 & 0.419 \\
\hline \multirow[t]{3}{*}{ HAT4 } & 0.5 & 9.25 & 1.04 & 0.660 \\
\hline & 0.1 & 9.25 & 1.95 & 0.801 \\
\hline & 0.02 & 9.25 & 3.91 & 1.2 \\
\hline \multirow[t]{3}{*}{ HAT5 } & 0.5 & 15.6 & 0.892 & 1.2 \\
\hline & 0.1 & 15.2 & 1.70 & 1.6 \\
\hline & 0.02 & 15.7 & 2.2 & 2.4 \\
\hline
\end{tabular}

Table II, and those at $C_{\mathrm{s}}=0.005,0.02,0.1,0.5$, and $2.5 \mathrm{M}$ are plotted double-logarithmically against $M_{w}$ in Figure 3. The data points at each $C_{\mathrm{s}}$ approximately follow a straight line, whose slope is, for example, 0.79 at $C_{\mathrm{s}}=2.5$ and 1.0 at $C_{\mathrm{s}}=0.005 \mathrm{M}$. These viscosity exponents are somewhat smaller than those $(0.84$ and 1.14 at 2.5 and $0.005 \mathrm{M}$, respectively) estimated previously $^{6}$ in the $M_{w}$ region between $10^{4}$ and $3.5 \times 10^{5}$, suggesting that the $\log [\eta] v s . \log M_{w}$ relations for $M_{w}>10^{4}$ have a weak downward curvature in the $C_{\mathrm{s}}$ range studied. 
Table II. Intrinsic viscosities for sodium hyaluronate samples in aqueous $\mathrm{NaCl}$ with different salt concentrations at $25^{\circ} \mathrm{C}$

\begin{tabular}{|c|c|c|c|c|c|c|c|c|c|c|}
\hline \multirow{3}{*}{ Sample } & \multicolumn{10}{|c|}{$[\eta] \times 10^{-2} / \mathrm{cm}^{3} \mathrm{~g}^{-1}$} \\
\hline & \multicolumn{10}{|c|}{$C_{\mathrm{s}} / \mathrm{M}$} \\
\hline & 0.005 & 0.0067 & 0.01 & 0.02 & 0.05 & 0.1 & 0.2 & 0.5 & 1.0 & 2.5 \\
\hline HAT1 & 11.5 & 10.5 & 9.19 & 7.40 & 5.92 & 4.97 & 4.33 & 3.96 & 3.66 & 3.33 \\
\hline HAT2 & 14.8 & 13.3 & 11.6 & 9.34 & 7.18 & 6.22 & 5.52 & 4.95 & 4.65 & 4.24 \\
\hline HAT3 & 26.4 & 23.7 & 20.0 & 15.7 & 12.8 & 10.8 & 9.61 & 8.05 & 7.40 & 6.74 \\
\hline HAT4 & 59.2 & 52.8 & 43.4 & 33.1 & 24.8 & 20.7 & 17.5 & 14.7 & 13.3 & 11.9 \\
\hline HAT5 & 93.8 & 86.3 & 68.8 & 52.1 & 40.1 & 32.5 & 27.7 & 22.9 & 20.6 & 18.3 \\
\hline
\end{tabular}

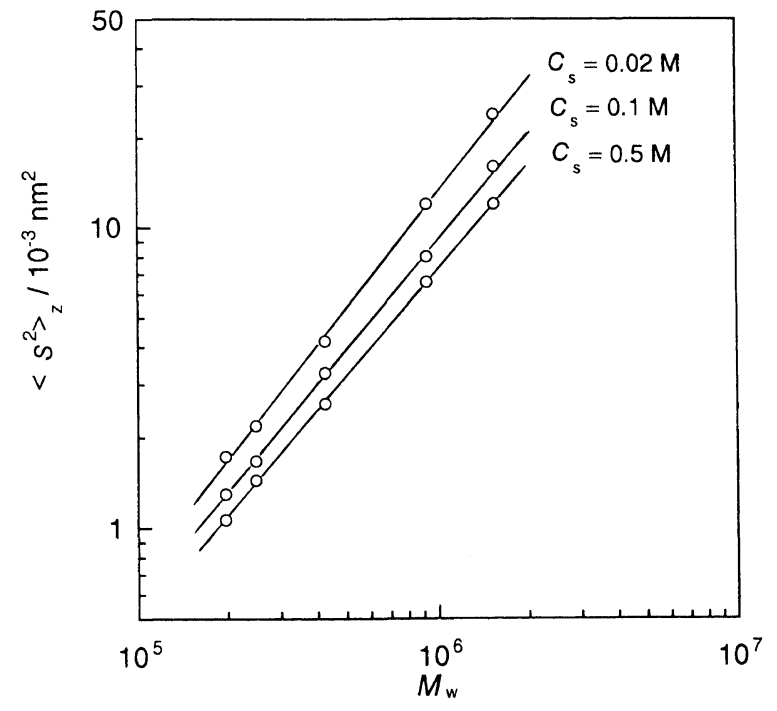

Figure 2. Molecular weight dependence of $\left\langle S^{2}\right\rangle_{z}$ for Na hyaluronate in aqueous $\mathrm{NaCl}$ with $C_{\mathrm{s}}=0.02,0.1$, and $0.5 \mathrm{M}$ at $25^{\circ} \mathrm{C}$.

\section{DISCUSSION}

Intrinsic Viscosity

In the QTP scheme ${ }^{3,11,12}$ for nonionic wormlike or helical wormlike bead chains, the viscosity expansion factor $\alpha_{\eta}\left[=\left([\eta] /[\eta]_{0}\right)^{1 / 3}\right]$ is a function only of the scaled excluded-volume parameter $\tilde{z}$ defined by

$$
\tilde{z}=(3 / 4) K(\lambda L) \mathrm{z}
$$

with

$$
z=(3 / 2 \pi)^{3 / 2}(\lambda B)(\lambda L)^{1 / 2}
$$

and

$$
\begin{aligned}
K(\lambda L)= & (4 / 3)-2.711(\lambda L)^{-1 / 2}+(7 / 6)(\lambda L)^{-1} \text { for } \lambda L>6 \\
= & (\lambda L)^{-1 / 2} \exp \left[-6.611(\lambda L)^{-1}\right. \\
& +0.9198+0.03516 \lambda L] \text { for } \lambda L<6
\end{aligned}
$$

Here, $[\eta]_{0}$ is the unperturbed intrinsic viscosity, $\lambda^{-1}$ is the stiffness parameter, $L$ is the contour length of the chain related to the molecular weight $M$ by $M_{L}=M / L$, with $M_{L}$ being the molar mass per unit contour length, $z$ is the conventional excluded-volume parameter, and $B$ is the excluded-volume strength for the interaction between a pair of beads. For the wormlike chain (a special limit of the helical wormlike chain) we have ${ }^{3}$

$$
\begin{aligned}
& \lambda^{-1}=2 q \\
& B=\beta / a^{2}
\end{aligned}
$$

where $q$ is the persistence length and $\beta$ the binary cluster

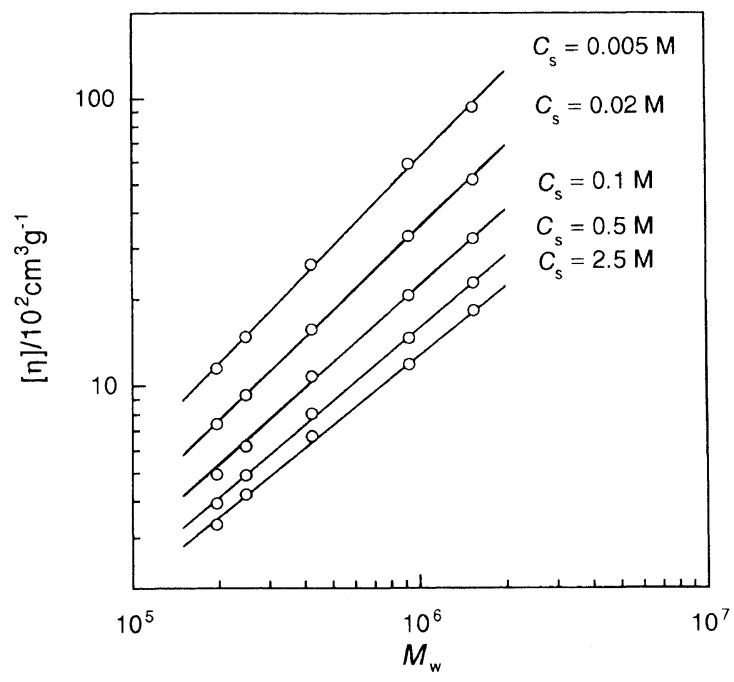

Figure 3. Molecular weight dependence of $[\eta]$ for $\mathrm{Na}$ hyaluronate in aqueous $\mathrm{NaCl}$ with indicated $C_{\mathrm{s}}$ at $25^{\circ} \mathrm{C}$.

integral, and $a$ the bead spacing; to be consistent with the notation in our previous work, $q$ is used hereafter instead of $\lambda^{-1}$. If the Barrett function ${ }^{13}$ is adopted, $\alpha_{\eta}^{3}$ in the QTP scheme reads

$$
\alpha_{\eta}^{3}=\left(1+3.8 \tilde{z}+1.9 \tilde{z}^{2}\right)^{0.3}
$$

The Yamakawa-Fujii-Yoshizaki theory ${ }^{7,8}$ for $[\eta]_{0}$ of an unperturbed wormlike chain contains $M_{L}, q$, and $d$ (the hydrodynamic diameter in the cylinder model) as the parameters, so that $[\eta]$ for a given $M$ in a perturbed state is determined by $M_{L}, q, d$, and $B$; note that this theory is essentially equivalent to that ${ }^{14}$ based on the touched-bead model if the difference in hydrodynamic diameter is taken into account.

Hayashi et al $^{5,6}$ estimated all the parameters except $d$ as a function of $C_{\mathrm{s}}$ from their [ $\left.\eta\right]$ data for $M_{w}$ between $3.8 \times 10^{3}$ and $3.5 \times 10^{5}$; they determined $d$ to be $1.0 \mathrm{~nm}$ at $C_{\mathrm{s}}=0.2 \mathrm{M}$ with the aid of Cleland's data ${ }^{15}$ for oligomers. Assuming, as in the work of Hayashi et al., that this $d$ value is applicable to any other salt concentrations, we re-estimated $q$ and $B$ at each $C_{\mathrm{s}}$ in such a way that for the previous $M_{L}$ value, ${ }^{5,6}$ the Yamakawa-Fujii-Yoshizaki theory combined with eq 6 most closely fits the previous and present $[\eta]$ data; we note that Hayashi et al. determined $M_{L}$ using data for low $M_{w}$, and also that excluded-volume effects on [ $\left.\eta\right]$ become appreciable almost regardless of $C_{\mathrm{s}}$ when $M_{w}$ exceeds $1.5 \times 10^{4} .{ }^{6}$ Our analysis showed that only $B$ values at several salt concentrations need to be slightly altered. The results are given in Tabie III, along with the previous $q$ and $M_{L}$ data (see ref 6 for discussions on 
Table III. Wormlike chain parameters and excluded-volume strength for sodium hyaluronate in aqueous $\mathrm{NaCl}$ at $25^{\circ} \mathrm{C}$

\begin{tabular}{|c|c|c|c|}
\hline$C_{\mathrm{s}}$ & $M_{L}^{a}$ & $q^{a}$ & $B$ \\
\hline M & $\mathrm{nm}^{-1}$ & $\mathrm{~nm}$ & $\mathrm{~nm}$ \\
\hline 0.005 & 400 & 9.7 & $19(16)^{\mathrm{a}}$ \\
\hline 0.0067 & 400 & 8.5 & $17(14)^{\mathbf{a}}$ \\
\hline 0.01 & 400 & 7.4 & $13(11)^{\mathrm{a}}$ \\
\hline 0.02 & 405 & 6.0 & 9.0 \\
\hline 0.05 & 410 & 5.0 & $7.0(6.0)^{\mathrm{a}}$ \\
\hline 0.1 & 410 & 4.8 & 4.8 \\
\hline 0.2 & 405 & 4.2 & $4.0(3.4)^{\mathrm{a}}$ \\
\hline 0.5 & 400 & 4.1 & 2.8 \\
\hline 1 & 400 & 4.0 & 2.3 \\
\hline 2.5 & 405 & 4.0 & 1.6 \\
\hline
\end{tabular}

a Previous estimates.

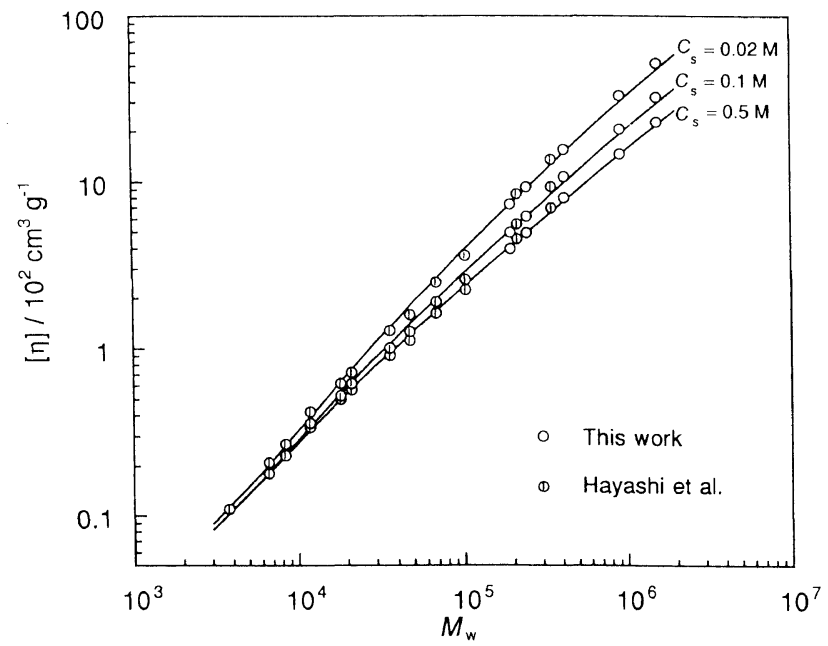

Figure 4. Comparison between the experimental $[\eta]$ at the indicated $C_{\mathrm{s}}$ (the circles) and the theoretical curves computed from the Yamakawa-Fujii-Yoshizaki theory ${ }^{7,8}$ for unperturbed wormlike chains and eq 6 with $d=1.0 \mathrm{~nm}$ and the parameters in Table III. (O), this work; (D), Hayashi et al. ${ }^{5,6}$

the $C_{\mathrm{s}}$ dependence of $q$ or the electrostatic persistence length ${ }^{16,17}$ ).

Figure 4 compares the best fit theoretical curves with the $[\eta]$ data at $C_{\mathrm{s}}=0.02,0.1$, and $0.5 \mathrm{M}$. The agreement is generally good throughout the entire range of molecular weight. Similar degrees of agreement were found at the other salt concentrations higher than $0.0067 \mathrm{M}$. However, close examination of Figure 4 shows that the curves deviate slightly upward for $M_{w}$ between $3 \times 10^{4}$ and $1.5 \times 10^{5}$ and downward for $M_{w}$ above $3 \times 10^{5}$. The deviation appears to become larger with lowering $C_{\mathrm{s}}$.

The comparison at the lowest $C_{\mathrm{s}}$ of $0.005 \mathrm{M}$ is illustrated in Figure 5, where the solid and dashed lines represent the theoretical $[\eta]$ and $[\eta]_{0}$, respectively. While, as is the case at higher $C_{\mathrm{s}}$, the experimental relation is essentially linear over a range of $M_{w}$ from $1 \times 10^{4}$ to $2 \times 10^{5}$, the solid curve here has a more pronounced downward curvature for $M>3 \times 10^{4}$. This difference results in an appreciable upward deviation of the solid curve (for the chosen parameters) from the data points in the $M_{w}$ range between $3 \times 10^{4}$ and $1.5 \times 10^{5}$, the deviation amounting to $10-20 \%$ (almost twice those at $C_{\mathrm{s}}>0.01 \mathrm{M}$ ). The theoretical exponent $v_{\alpha}^{3}$ for $\alpha_{\eta}^{3}$ in this $M$ range is 0.25 , whereas the corresponding experimen-

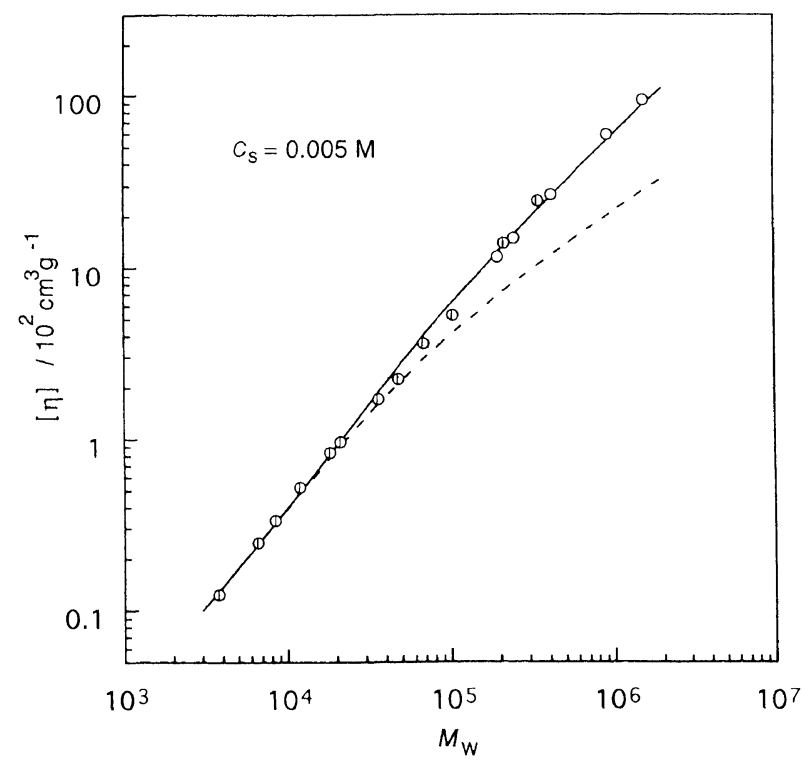

Figure 5. Comparison between the experimental $[\eta]$ at $C_{\mathrm{s}}=0.005 \mathrm{M}$ (the circles) and the theoretical curve (the solid line) computed from the Yamakawa-Fujii-Yoshizaki theory ${ }^{7,8}$ for unperturbed wormlike chains and eq 6 with $d=1.0 \mathrm{~nm}$ and the parameters in Table III. The dashed line represents the theoretical values in the unperturbed state $(B=0)$. The symbols are the same as those used in Figure 4.

tal $v_{\alpha}^{3}$ value ( $=$ the difference between the experimental exponent of 1.14 for $[\eta]$ and the theoretical exponent of 0.79 for $\left.[\eta]_{0}\right)$ is as large as 0.35 , i.e., a value that exceeds the asymptotic exponent 0.30 predicted by eq 6 . Note that choice of a smaller $B$ leads to a smaller $v_{\alpha}^{3}$ and a much larger discrepancy between the theoretical and experimental $[\eta]$ at high $M_{w}$.

The QTP theory uses the pseudo (or short-range) potential for the excluded-volume interaction between a pair of beads. This approximation may be considered valid even for polyelectrolytes at low ionic strength if the chains are sufficiently long compared to the range that electrostatic interactions between charged beads extend. In other words, the QTP approximation to $\alpha_{\eta}^{3}$ for $\mathrm{Na}$ hyaluronate should be better for higher $M$. Interestingly, when $M_{w}$ exceeds $2 \times 10^{5}$ (Figure 5), such discrepancy in $v_{\alpha}^{3}$ as mentioned above almost disappears or at least considerably diminishes owing to the decrease in experimental exponent for $[\eta]$. This finding suggests that the observed poor agreement in $[\eta]$, at least, at $C_{\mathrm{s}}<$ $0.01 \mathrm{M}$ has something to do with the short-range potential used in the QTP theory. Of course, to ascertain this, we need theoretical work on a chain with both non-electrostatic short-range and electrostatic longrange (Coulomb) potentials.

Although the discrepancies between theory and experiment observed in Figure 4 are somewhat systematic, they are as small as what has been observed for nonionic semiflexible polymers. ${ }^{2}$ A point to note is that for those polymers including $\mathrm{Na}$ hyaluronate, small theoretical errors may come from the estimated wormlike chain parameters. In view of these, it is probably fair to say that the QTP theory combined with the Yamakawa-Fujii-Yoshizaki theory has no serious shortcomings at $C_{\mathrm{s}} \geq 0.01 \mathrm{M}$.

\section{Radius of Gyration}

The radius expansion factor $\alpha_{\mathrm{s}}\left[=\left(\left\langle S^{2}\right\rangle /\left\langle S^{2}\right\rangle_{0}\right)^{1 / 2}\right]$ is 


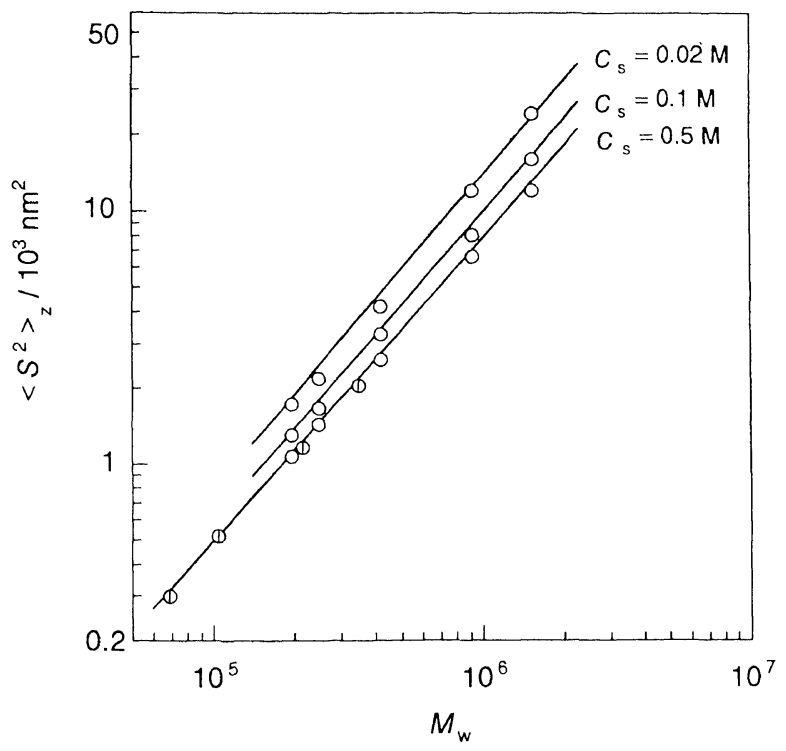

Figure 6. Comparison between the experimental $\left\langle S^{2}\right\rangle_{z}$ at the indicated $C_{\mathrm{s}}$ and the theoretical curves calculated from eq 7 and 8 with the parameters in Table III. The symbols are the same as those used in Figure 4.

also a function only of $\tilde{z}$ in the QTP scheme, where $\left\langle S^{2}\right\rangle_{0}$ (the unperturbed mean-square radius of gyration) for the wormlike chain is given by ${ }^{18}$

$$
\begin{aligned}
\left\langle S^{2}\right\rangle_{0}= & (q L / 3)-q^{2}+\left(2 q^{3} / L\right) \\
& -\left(2 q^{4} / L^{2}\right)[1-\exp (-L / q)]
\end{aligned}
$$

and $\alpha_{\mathrm{s}}^{2}$ is expressed by

$$
\begin{aligned}
\alpha_{\mathrm{s}}^{2}= & {\left[1+10 \tilde{z}+\left(\frac{70 \pi}{9}+\frac{10}{3}\right) \tilde{z}^{2}+8 \pi^{3 / 2} \tilde{z}^{3}\right]^{2 / 15} } \\
& \times\left[0.933+0.067 \exp \left(-0.85 \tilde{z}-1.39 \tilde{z}^{2}\right)\right]
\end{aligned}
$$

if the Domb-Barrett function ${ }^{19}$ is used. For large $L / q$ with which the present analysis is concerned, $\left\langle S^{2}\right\rangle_{0}$ is determined substantially by the first two leading terms of eq 7 , so that its $z$-average $\left(\left\langle S^{2}\right\rangle_{0 z}\right)$ may be evaluated by use of the $z$-average $L$, i.e., $M_{z} / M_{L}$, with $M_{z}$ being the $z$-average molecular weight.

In Figure 6, the theoretical curves computed from eq 7 and 8 with the parameters in Table III are compared with our $\left\langle S^{2}\right\rangle_{z}$ data at $C_{\mathrm{s}}=0.02,0.1$, and $0.5 \mathrm{M}$ and those of Hayashi et al. ${ }^{5}$ at $0.5 \mathrm{M}$, where $\left\langle S^{2}\right\rangle_{0}$ has been replaced by $\left\langle S^{2}\right\rangle_{0 z}$ calculated with an $M_{z} / M_{w}$ of 1.1 , the average obtained previously from sedimentation equilibrium. ${ }^{5}$ All the curves come close to the data points for the respective salt concentrations; though the slope 1.21 of the line for $C_{\mathrm{s}}=0.02 \mathrm{M}$ is appreciably smaller than that (1.26) of the corresponding experimental relation, the differences between the theoretical and experimental $\left\langle S^{2}\right\rangle_{z}$ values are very small in the molecular weight range studied here. Hence, we may conclude that with the parameters derived from $[\eta]$, the combination of eq 7 and 8 describes the present $\left\langle S^{2}\right\rangle_{z}$ data for $\mathrm{Na}$ hyaluronate in the three aqueous salts.

As Table III shows, the (total) persistence length (estimated from $[\eta]$ ) increases remarkably with decreasing $C_{\mathrm{s}}$. This implies that enhancement of electrostatic repulsion between neighboring charged groups of the hyaluronate chain leads to a considerable increase in $\left\langle S^{2}\right\rangle_{0}$. Very recently, $\mathrm{Na}$ hyaluronate samples of low molecular weight $\left(M_{w}=3.8 \times 10^{3}-1.1 \times 10^{4}\right)$ in 0.02 and $0.1 \mathrm{M}$ aqueous $\mathrm{NaCl}$ at $25^{\circ} \mathrm{C}$ were studied by small-angle $\mathrm{X}$-ray scattering in our laboratory, ${ }^{19}$ and it was found that the measured $\left\langle S^{2}\right\rangle_{z}$ and scattering functions (in the angular range examined) are almost quantitatively explained by the unperturbed wormlike chain with the $q$ and $M_{L}$ values in Table III. This confirms the unperturbed dimensions of the polysaccharide in the two aqueous salts estimated indirectly from $[\eta]$. Thus, the agreement observed in Figure 6 can be taken to show that eq 8 is a good approximation to the charged polysaccharide in $0.02,0.1$, and $0.5 \mathrm{M}$ aqueous $\mathrm{NaCl}$.

\section{CONCLUSIONS}

We have examined the applicability of QTP theory ${ }^{3}$ for nonionic chains to $\left\langle S^{2}\right\rangle$ and $[\eta]$ of $\mathrm{Na}$ hyaluronate, a charged polysaccharide, in aqueous $\mathrm{NaCl}$ with the aid of relevant theories ${ }^{7,8,18}$ for unperturbed wormlike chains. The agreement between the theoretical and experimental $[\eta]$ becomes poor as $C_{\mathrm{s}}$ is lowered, but the QTP theory is a fairly satisfactory approximation to both $\left\langle S^{2}\right\rangle$ and $[\eta]$ of the polysaccharide unless $C_{\mathrm{s}}$ is lower than $0.02 \mathrm{M}$. Below $C_{\mathrm{s}}=0.01 \mathrm{M}$, this theory combined with the Yamakawa-Fujii-Yoshizaki theory ${ }^{7,8}$ fails to explain the experimental viscosity exponents in a limited range of molecular weight from $3 \times 10^{4}$ to $1.5 \times 10^{5}$. This failure is tentatively attributed to the short-range potential used in the QTP theory for excluded-volume interactions between beads.

Acknowledgments. We thank Dr. T. Yanaki of Shiseido Co. for providing a hyaluronate sample. This work was supported in part by a Grant-in-Aid (09450363) for Scientific Research from the Ministry of Education, Science, Sports, and Culture of Japan.

\section{REFERENCES}

1. See, for flexible polymers, F. Abe, Y. Einaga, T. Yoshizaki, and H. Yamakawa, Macromolecules, 26, 1884 (1993); K. Horita, F. Abe, Y. Einaga, and H. Yamakawa, Macromolecules, 26, 5067 (1993); F. Abe, K. Horita, Y. Einaga, and H. Yamakawa, Macromolecules, 27, 725 (1994); M. Kamijo, F. Abe, Y. Einaga, and H. Yamakawa, Macromolecules, 28, 1095 (1995).

2. See, for stiff chains, T. Norisuye, A. Tsuboi, and A. Teramoto, Polym. J., 28, 357 (1996).

3. H. Yamakawa, "Helical Wormlike Chains in Polymer Solutions," Springer, Berlin, 1997.

4. O. Kratky and G. Porod, Rec. Trav. Chim., 68, 1106 (1949).

5. K. Hayashi, K. Tsutsumi, F. Nakajima, T. Norisuye, and A. Teramoto, Macromolecules, 28, 3824 (1995)

6. K. Hayashi, K. Tsutsumi, T. Norisuye, and A. Teramoto, Polym. J., 28, 922 (1996).

7. H. Yamakawa and M. Fujii, Macromolecules, 7, 128 (1974).

8. H. Yamakawa and T. Yoshizaki, Macromolecules, 13, 633 (1980).

9. Y. Nakanishi, T. Norisuye, A. Teramoto, and S. Kitamura, Macromolecules, 26, 4220 (1993)

10. H. Fujita, "Polymer Solutions," Elsevier, Amsterdam, 1990.

11. H. Yamakawa and W. H. Stockmayer, J. Chem. Phys., 57, 2843 (1972).

12. J. Shimada and H. Yamakawa, J. Chem. Phys., 85, 591 (1986).

13. A. J. Barrett, Macromolecules, 17, 1566 (1984).

14. T. Yoshizaki, I. Nitta, and H. Yamakawa, Macromolecules, 21, 165 (1988).

15. R. L. Cleland, Biopolymers, 23, 647 (1984).

16. T. Odijk, J. Polym. Sci., Polym. Phys. Ed., 15, 477 (1977).

17. J. Skolnick and M. Fixman, Macromolecules, 10, 944 (1977).

18. H. Benoit and P. Doty, J. Phys. Chem., 57, 958 (1953).

19. C. Domb and A. J. Barrett, Polymer, 17, 179 (1976).

20. N. Mizukoshi and T. Norisuye, to be published. 